\title{
Análise da Qualidade e Estilo de Vida entre Acadêmicos de Medicina de uma Instituição do Norte do Paraná
}

\section{Analysis of Quality and Lifestyle among Medical Students of an Institution in the North of Paraná}

\section{PALAVRAS-CHAVE \\ - Educação Superior. \\ - Estilo de Vida. \\ - Estudantes de Medicina. \\ - Saúde.}

Bruna Elisa Bührer ${ }^{I}$ Ana Carolina Tomiyoshi ${ }^{I}$ Marcela Demitto Furtado ${ }^{I I}$ Fernanda Shizue Nishida ${ }^{I, I I I}$

\begin{abstract}
RESUMO
O objetivo do estudo foi avaliar o estilo de vida e estimar a prevalência do consumo de álcool, tabaco e outras drogas entre estudantes de Medicina de uma instituição de Maringá, no Paraná. Trata-se de um estudo transversal, descritivo, exploratório realizado com universitários do primeiro ao sexto ano do curso de Medicina. A coleta de dados ocorreu de junho a agosto de 2017 por meio do instrumento autoaplicável denominado "Questionário de Estilo de Vida Fantástico". Composto por 25 questões objetivas, esse instrumento considera o comportamento dos indivíduos no último mês e seus resultados permitem determinar a associação entre estilo de vida e saúde. A soma de todos os pontos permite chegar a um escore total que classifica os indivíduos em cinco categorias: "excelente", "muito bom", "bom", "regular" e "necessita melhorar". É desejável que os indivíduos atinjam a classificação "bom". Quanto menor o escore, maior será a necessidade de mudança. Os critérios de exclusão adotados foram: estudantes menores de 18 anos e aqueles que não estivessem presentes no dia da coleta. Após a coleta, os dados foram transcritos para uma planilha eletrônica e posteriormente analisados utilizando-se estatística descritiva. Participaram da pesquisa 576 acadêmicos, dos quais a maioria com idade entre 21 e 25 anos, sexo feminino, cor/raça branca, solteiros, estudaram em escola privada no ensino médio e moram sozinhos. Em relação à escolaridade do pai e da mãe, observou-se que $73,61 \%$ e 83,68\% estudaram por 12 ou mais anos, respectivamente. Menos da metade dos estudantes apresentaram diagnóstico de depressão ou outra patologia crônica psiquiátrica. Grande parte dos alunos não dorme bem e não se sente descansada. Observou-se alta prevalência de estudantes que estão satisfeitos com seus trabalhos ou funções. A maioria referiu não ter fumado no último ano e nunca ter usado drogas como maconha e cocaína, enquanto $81 \%$ relataram uma ingestão média de álcool por semana de zero a sete doses. A classificação predominante no EVF foi "bom", e, com isso, conclui-se que os estudantes devem ser orientados a adotar um estilo de vida mais saudável, que se concilie com as atividades acadêmicas.
\end{abstract}




\section{KEY-WORDS}

- Higher Education.

- Lifestyle.

- Medical Student.

- Health.
ABSTRACT

The purpose of this research was to evaluate the lifestyle and estimate the prevalence of consumption of alcohol, tobacco and other drugs among medical students of an institution in Maringá, Paraná. It is a cross-sectional, exploratory, descriptive study, with undergraduates from the first to sixth years of medical school. The data collection was carried out from June to August 2017, using a selfadministered instrument called the "Fantastic Lifestyle Checklist", which is composed of 25 objective questions, that consider the individual's behavior in the last month. The results of the questionnaire are used to determine the association between lifestyle and health. Totalling all the points gives a total score that classifies individuals into one of five categories: "Excellent", "Very Good", "Good", "Fair" and "Needs improvement". It is desirable for individuals to achieve a "Good" rating. The lower the score, the greater the need for change. The exclusion criteria adopted were: students under 18 years old, and those not present on the day of the data collection. Next, the data were transferred to spreadsheet, then analyzed using descriptive statistics. 576 medical students participated in the research, the majority aged between 21 and 25 years old, female, White, single, had studied at a private high school, and living alone. Regarding the parents' level of education, the data showed that $73.61 \%$ of the fathers and $83.68 \%$ of the mothers had studied for 12 or more years. Less than half of the students reported a diagnosis of depression or other chronic mental health condition. Most of the students did not sleep well and did not feel rested. There was a high prevalence of students who are satisfied with their respective jobs or positions. In addition, most reported that they had not smoked in the last year and had never used drugs such as marijuana and cocaine, while $81 \%$ reported an average alcohol intake per week of 0 to 7 units. The predominant classification among the students in the Fantastic Lifestyle Questionnaire was "Good". It is therefore concluded that students should be given guidance on how to adopt a healthier lifestyle while engaged in academic activities.

Recebido em: 23/6/2018

Aceito em: 5/8/2018

\section{INTRODUÇÃO}

Atualmente, muito se tem estudado a saúde física e mental de estudantes de Medicina a fim de conhecer seus hábitos e estimar sua qualidade de vida para, assim, buscar prevenir agravos à sua saúde ${ }^{1}$. $\mathrm{O}$ acesso ao curso de Medicina geralmente ocorre em um clima de tensão e competitividade por ser um dos mais concorridos processos seletivos universitários. Portanto, a dedicação se inicia cedo, antes mesmo da graduação ${ }^{1,2}$.

O clima de tensão e sentimentos angustiantes podem piorar durante o curso médico, pois diversos fatores - como pressão para aprender grande quantidade de novas informações, pouco tempo para atividades de lazer, contato com a morte e doenças graves em idade precoce ${ }^{3,4}$-colaboram para o déficit da saúde mental e piora da qualidade de vida ${ }^{4,5}$. Qualidade de vida é definida pela forma humana de percepção do próprio existir, com base em esferas objetivas e subjetivas ${ }^{6}$. A esfera subjetiva de compreensão de qualidade de vida diz respeito ao estilo de vida do sujeito, relacionado com a realidade familiar, ambiental e social ${ }^{6,7}$. Já estilo de vida pode ser definido como um padrão de comportamento modificável e que pode ocasionar um efeito profundo na saúde dos seres humanos, pois se relaciona com diversos aspectos que se refletem na vida das pessoas ${ }^{8,9}$.

Durante a graduação, os acadêmicos podem sofrer prejuízos no domínio psicológico, o que irá trazer importantes implicações para a saúde do futuro médico. Segundo Costa et al. ${ }^{5}$, a elevada exaustão emocional causa uma adaptação inadequada às dificuldades, levando a atitudes de indiferença e insensibilidade. Esse sentimento rotineiro leva a uma sensação de fracasso, e as atividades perdem o sentido ${ }^{5}$. Em decorrência desse estresse e falta de motivação, alguns estudos mostram alta taxa de suicídio, depressão e uso de drogas em médicos e estudantes de Medicina, os quais podem afetar a relação médico-paciente ${ }^{10,11}$.

Culturalmente, a medicina é uma profissão de muito comprometimento com o próximo, pois lida com a vida e a morte em sua integridade ${ }^{12}$. No entanto, cuidar do outro requer cuidar de si. Assim, acredita-se que investigar aspectos relacionados ao estilo de vida de estudantes de Medicina é necessário para o desenvolvimento de ações de promoção da saúde neste grupo ${ }^{13}$, além de subsidiar a reflexão acerca da saúde do futuro profissional médico, evitando, assim, adoecimento e esgotamento ${ }^{12}$. 
Nesse sentido, o objetivo deste estudo foi avaliar o estilo de vida e estimar a prevalência do consumo de álcool, tabaco e outras drogas entre estudantes de Medicina de uma instituição de Maringá, no Paraná.

\section{METODOLOGIA}

Trata-se de um estudo transversal, descritivo, exploratório, realizado com os 835 alunos matriculados no curso de Medicina, desde a primeira até a sexta série, de uma instituição de ensino superior do município de Maringá, no Paraná.

A coleta de dados foi realizada entre os meses de junho e agosto de 2017 pelos pesquisadores, por meio de um questionário autoaplicável denominado "Estilo de Vida Fantástico" $(E V F)^{14,15}$, composto por 25 questões. Os pesquisadores aguardaram o preenchimento em sala.

O questionário EVF é um instrumento autoaplicável que considera o comportamento dos indivíduos no último mês e cujos resultados permitem associar estilo de vida e saúde ${ }^{14,15}$. As questões estão dispostas na forma de escala Likert. A soma de todos os pontos permite chegar a um escore total que classifica os indivíduos em cinco categorias: "excelente" (85 a 100 pontos), "muito bom" (70 a 84 pontos), "bom" (55 a 69 pontos), "regular" (35 a 54 pontos) e "necessita melhorar" (0 a 34 pontos). É desejável que os indivíduos atinjam a classificação "bom". Quanto menor for o escore, maior será a necessidade de mudança ${ }^{14,15}$.

A palavra "fantástico" vem do acrônimo fantastic, que representa as letras iniciais de nomes de nove domínios (na lín- gua inglesa) em que estão distribuídas as 25 questões ou itens: $\mathrm{F}=$ family and friends (família e amigos); $\mathrm{A}=$ activity (atividade física); $\mathrm{N}=$ nutrition (nutrição); $\mathrm{T}=$ tobacco $\mathcal{E}$ toxics (cigarro e drogas); $\mathrm{A}=$ alcohol (álcool); $\mathrm{S}=$ sleep, seatbelts, stress, safe sex (sono, cinto de segurança, estresse e sexo seguro); $\mathrm{T}=$ type of behavior (tipo de comportamento; padrão de comportamento A ou B); I = insight (introspecção); C = career (trabalho; satisfação com a profissão).

Os pesquisadores aplicaram o questionário ao final de algumas aulas, tendo sido previamente solicitada autorização da coordenação do curso de Medicina, bem como do professor responsável pela aula no momento da coleta. Como critério de inclusão, foram selecionados todos os estudantes de Medicina da instituição pesquisada, além de ser solicitado a eles que lessem e assinassem o Termo de Consentimento Livre e Esclarecido. Os critérios de exclusão adotados foram: estudantes menores de 18 anos, aqueles que não estavam presentes no dia da coleta e os alunos que não assinaram o Termo de Consentimento Livre e Esclarecido. Assim, a população do estudo foi composta por 576 alunos (Figura 1).

Após a coleta, os dados foram transcritos para uma planilha eletrônica do Excel e analisados por meio de estatística descritiva, sendo os dados apresentados no formato de tabelas. Esta pesquisa seguiu todas as recomendações da Resolução $n^{\circ}$ 466/2012 do Conselho Nacional de Saúde e foi aprovada pelo Comitê Permanente de Ética em Pesquisa Envolvendo Seres Humanos do Centro Universitário Cesumar, com Parecer $n^{\circ}$ 2.022.114, em 19 de abril de 2017.

Figura 1

Diagrama do número esperado de participantes e o número obtido após critérios de exclusão

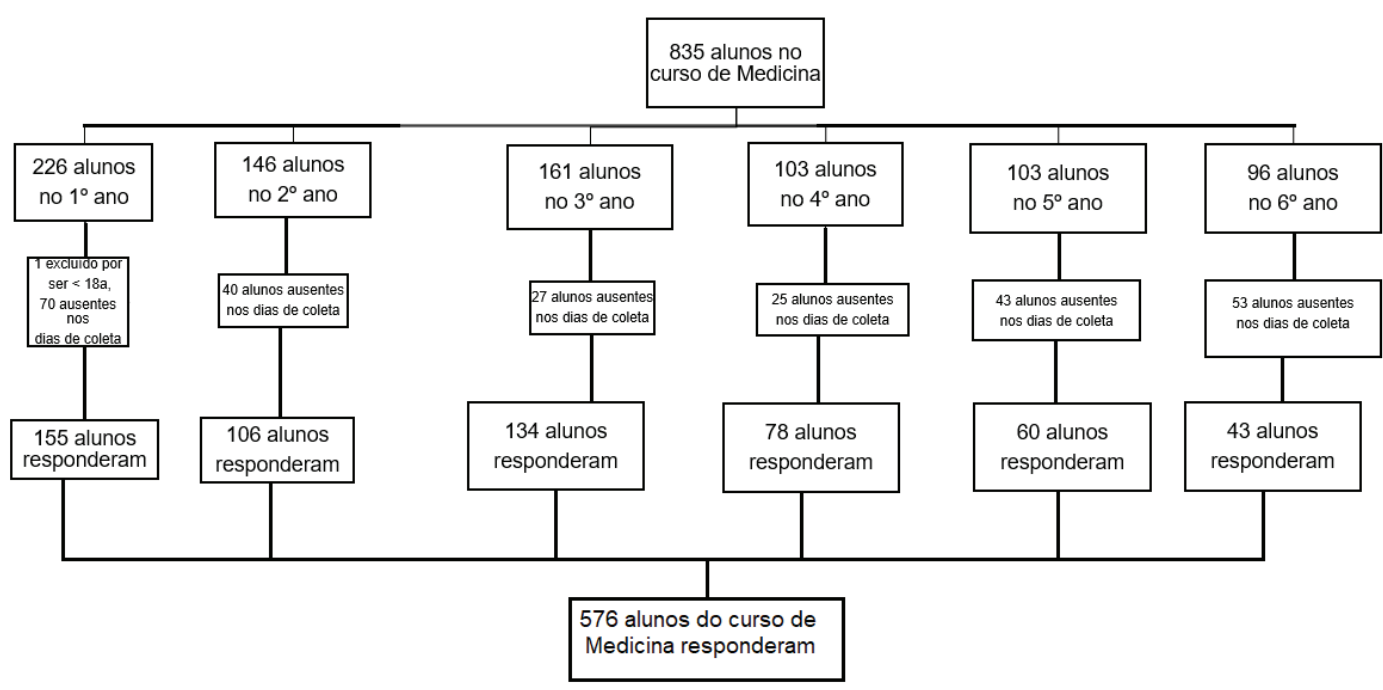

Fonte: Os autores. 


\section{RESULTADOS}

Dos 576 participantes da pesquisa, observou-se que a maioria possui idade entre 21 e 25 anos (58,51\%), é do sexo feminino (58,68\%) e de cor/raça branca $(86,63 \%)$. Apenas 7,47\% dos estudantes respondentes são da sexta série do curso, enquanto $26,91 \%$ são da primeira. A grande maioria deles é solteira $(95,31 \%)$, estudou em escola privada no ensino médio $(83,68 \%)$ e mora sozinha $(53,65 \%)$. Em relação à escolaridade do pai e da mãe, observa-se que 73,61\% e 83,68\% estudaram por 12 ou mais anos, respectivamente (Tabela 1 ).

Identificou-se que 28,65\% dos estudantes apontaram diagnóstico de depressão ou outra patologia crônica psiquiátrica, 26,56\% fazem uso de medicamentos psiquiátricos e 55,38\% relataram diagnóstico de depressão na família. Quanto à classificação dos acadêmicos em relação ao estilo de vida, $43,6 \%$ dos estudantes foram classificados como "bom", conforme demonstrado na Figura 2.

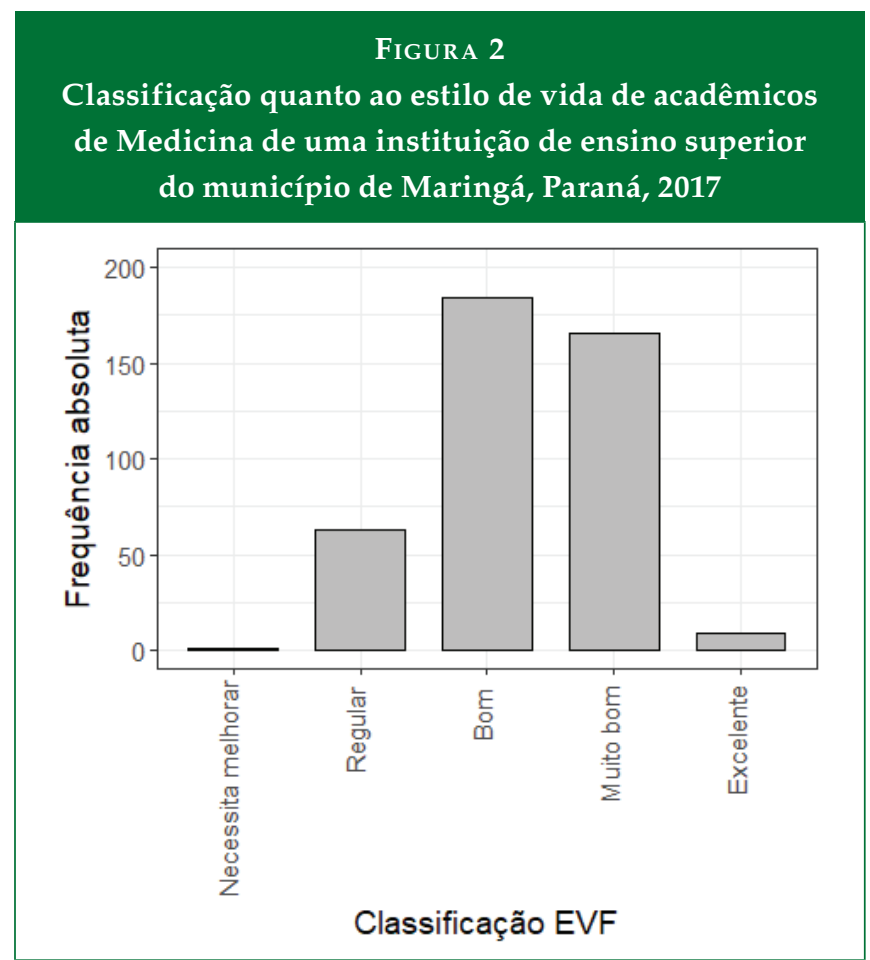

Fonte: Os autores.

Observou-se que os participantes da segunda série do curso de Medicina da IES pesquisada apresentam a maior prevalência na classificação "regular" em relação às outras séries do curso. Isso pode ser explicado pela baixa pontuação em vários itens do questionário aplicado, como "Sou vigorosamente ativo", "Sou moderadamente ativo", "Como uma dieta balanceada", "Consumo numa ocasião", "Durmo bem", "Sou capaz de lidar com meu estresse", "Relaxo no tempo de lazer", "Apre-

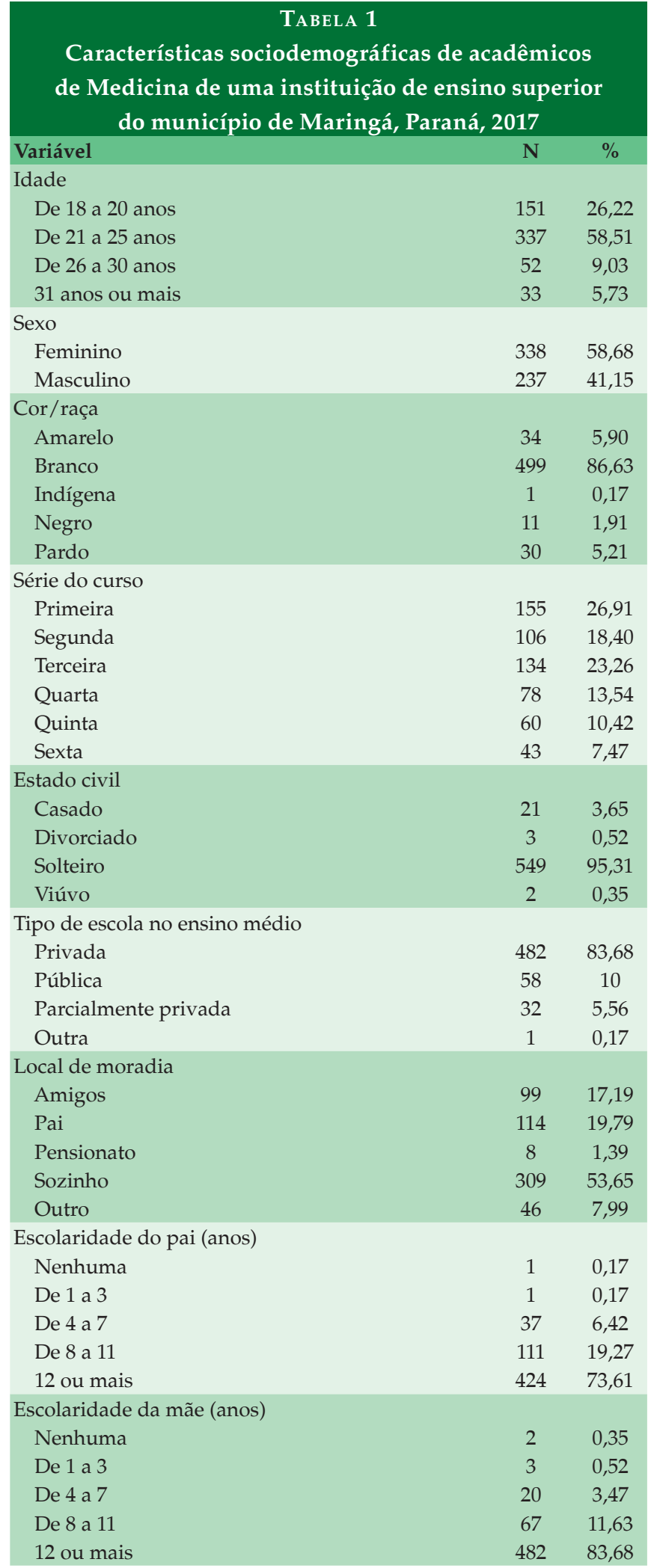

* As colunas não totalizam 100\%, já que alguns alunos deixaram questões sem resposta.

Fonte: Os autores. 
sento estar com pressa", "Sinto-me com raiva e hostil", "Penso de forma positiva", "Sinto-me tenso e desapontado", "Sinto-me triste e deprimido" e "Estou satisfeito com meu trabalho". Isso significa que os acadêmicos desta série ficaram com os menores escores em mais da metade das considerações do questionário.

A quarta série é a mais prevalente na classificação "excelente", seguida pela quinta e sexta séries. Os itens que receberam maior pontuação estão relacionados com boas práticas de saúde e são: "Dou e recebo afeto", "Sou vigorosamente ativo", "Sou moderadamente ativo", "Peso corporal saudável", "Fumo cigarro", "Uso drogas", "Abuso de remédios", "Bebo chá, café e colas", "Durmo bem", "Sou capaz de lidar com meu estresse", "Relaxo no tempo de lazer", "Penso de forma positiva", "Sinto-me tenso e desapontado", "Sinto-me triste e deprimido", "Estou satisfeito com meu trabalho" (Tabela 2).

\begin{tabular}{|c|c|c|c|c|c|c|c|c|c|c|c|c|c|c|}
\hline $\begin{array}{r}\text { Dist } \\
\text { escal } \\
\text { acadêmi } \\
\text { supe }\end{array}$ & & e & $\begin{array}{l}0 \\
\mathrm{~d} \\
\mathbf{N}\end{array}$ & $\begin{array}{l}\text { fre } \\
\text { lici }\end{array}$ & $\begin{array}{l}\text { TA } \\
\text { qu } \\
\text { do } \\
\text { na }\end{array}$ & $\begin{array}{l}B E L \\
\text { ênc }\end{array}$ & & 3 & 5 & sifi & & & - & \\
\hline & & & & & & Érie do & cur & & & & & & & lota \\
\hline $\begin{array}{l}\text { Classi } \\
\text { EVF }\end{array}$ & Prir & neira & & unda & & ceira & & arta & Qu & inta & & exta & & otal \\
\hline & $\mathrm{n}$ & $\%$ & $\mathrm{n}$ & $\%$ & $\mathrm{n}$ & $\%$ & $\mathrm{n}$ & $\%$ & $\mathrm{n}$ & $\%$ & $\mathrm{n}$ & $\%$ & $\mathrm{n}$ & \\
\hline Excelente & 1 & 0,2 & 1 & 0,2 & 0 & 0,0 & 3 & 0,7 & 2 & 0,5 & 2 & 0,5 & 9 & \\
\hline Muito bom & 38 & 8,8 & 23 & 5,3 & 45 & 10,4 & 26 & 6,0 & 25 & 5,8 & 11 & 2,5 & 168 & 38,8 \\
\hline Bom & 39 & 9,0 & 39 & 9,0 & 55 & 12,7 & 29 & 6,7 & 18 & 4,2 & 9 & 2,1 & 189 & 43,6 \\
\hline Regular & 19 & 4,4 & 22 & 5,1 & 15 & 3,5 & 1 & 0,2 & 5 & 1,2 & 4 & 0,9 & 66 & 15,2 \\
\hline $\begin{array}{l}\text { Necessita } \\
\text { melhorar }\end{array}$ & 0 & , & 1 & 2 & 0 & 0,0 & 0 & 0,0 & 0 & 0,0 & 0 & 0 & 1 & \\
\hline
\end{tabular}

Fonte: Os autores.

Observou-se que metade dos universitários apresenta dieta balanceada "quase nunca" a apenas "algumas vezes". Em relação a isso, verificou-se que 367 participantes da pesquisa fazem "uso de dois a quatro itens em excesso", que são açúcar, sal, gordura animal e bobagens e salgadinhos.

Analisou-se que $68 \%$ dos estudantes relataram que "não fumaram nenhum cigarro no ano passado", enquanto $2 \%$ e $7 \%$ fumam mais de dez e de um a dez cigarros por dia, respectivamente. Nota-se também que $74 \%$ dos acadêmicos nunca utilizaram drogas como maconha e cocaína, e a ingestão média de álcool por semana é de zero a sete doses em $81 \%$ dos entrevistados. Entretanto, 41\% dirigem após a ingestão de bebidas alcóolicas (Tabela 3).

Poucos alunos (13\%) dormem bem e se sentem descansados "quase sempre" e mais da metade (56\%) referem relaxar "algumas vezes" a "quase nunca" em seu tempo de lazer. Quanto ao trabalho, vê-se que 36\% estão "com relativa frequência" e 32\% "quase sempre" satisfeitos com os respectivos trabalhos ou funções. Grande parte dos alunos (35\%) declarou "Ser vigorosamente ativo" menos de uma vez por semana.
TABela 3

Distribuição de frequências e medidas descritivas das respostas aos itens da escala EVF entre acadêmicos de Medicina de uma instituição de ensino superior do município de Maringá, Paraná, 2017

\begin{tabular}{|c|c|c|c|c|c|c|}
\hline \multirow{2}{*}{ Itens da escala } & \multicolumn{6}{|c|}{ Frequência absoluta (\%) } \\
\hline & 0 & 1 & 2 & 3 & $4^{*}$ & Total $^{* *}$ \\
\hline 1 Tenho alguém para conversar & 1 & 1 & 10 & 17 & 70 & 99 \\
\hline 2 Dou e recebo afeto & 1 & 2 & 12 & 21 & 63 & 98 \\
\hline 3 Sou vigorosamente ativo & 35 & 18 & 22 & 12 & 11 & 98 \\
\hline 4 Sou moderadamente ativo & 27 & 27 & 14 & 11 & 15 & 93 \\
\hline 5 Como uma dieta balanceada & 10 & 10 & 30 & 32 & 15 & 97 \\
\hline 6 Excesso de açúcar, sal, gordura & 16 & 15 & 33 & 24 & 10 & 98 \\
\hline 7 Peso corporal saudável & 12 & 5 & 11 & 18 & 55 & 100 \\
\hline 8 Fumo cigarro & 2 & 7 & 12 & 4 & 68 & 93 \\
\hline 9 Uso drogas & 21 & 0 & 0 & 0 & 74 & 95 \\
\hline 10 Abuso de remédios & 1 & 3 & 8 & 26 & 61 & 99 \\
\hline 11 Bebo café, chá e colas & 3 & 2 & 25 & 58 & 12 & 99 \\
\hline 12 Consumo por semana & 2 & 3 & 4 & 9 & 81 & 98 \\
\hline 13 Consumo numa ocasião & 1 & 15 & 38 & 25 & 20 & 99 \\
\hline 14 Dirijo após beber & 41 & 0 & 0 & 0 & 55 & 97 \\
\hline 15 Durmo bem & 12 & 12 & 37 & 26 & 13 & 100 \\
\hline 16 Uso cinto de segurança & 0 & 2 & 4 & 14 & 79 & 99 \\
\hline $\begin{array}{l}17 \text { Sou capaz de lidar com meu } \\
\text { estresse }\end{array}$ & 4 & 7 & 27 & 40 & 21 & 99 \\
\hline 18 Relaxo no tempo de lazer & 9 & 13 & 34 & 24 & 19 & 100 \\
\hline 19 Pratico sexo seguro & 10 & 3 & 12 & 20 & 49 & 95 \\
\hline 20 Apresento estar com pressa & 26 & 26 & 32 & 11 & 3 & 99 \\
\hline 21 Sinto-me com raiva e hostil & 6 & 15 & 39 & 26 & 14 & 100 \\
\hline 22 Penso de forma positiva & 5 & 9 & 30 & 36 & 20 & 99 \\
\hline 23 Sinto-me tenso e desapontado & 8 & 20 & 44 & 20 & 8 & 100 \\
\hline 24 Sinto-me triste e deprimido & 5 & 12 & 35 & 32 & 16 & 100 \\
\hline $\begin{array}{l}25 \text { Estou satisfeito com meu } \\
\text { trabalho }\end{array}$ & 2 & 8 & 20 & 36 & 32 & 99 \\
\hline
\end{tabular}

* Os valores 0, 1, 2, 3 e 4 referem-se à frequência do hábito em cada pergunta, dependendo do hábito pesquisado para ser um aspecto positivo ou negativo no estilo de vida. Nas questões 1, 2, 5, 15, 17, 18, 22 e 25, os valores 0, 1, 2, 3 e 4 referem-se a "quase nunca", "raramente", "algumas vezes", "com relativa frequência" e "quase sempre", respectivamente. Na questão 3, 0, 1, 2, 3 e 4 significam "menos de 1 vez por semana", "1-2 vezes por semana", "3 vezes por semana", " 4 vezes por semana" $e$ " 5 ou mais vezes por semana", respectivamente. Na questão 4, os valores significam "menos de 1 vez por semana", "1-2 vezes por semana", " 3 vezes por semana", "4 vezes por semana" $e$ " 5 ou mais vezes por semana", nessa ordem. Os valores da questão 6 representam: "quatro itens", três itens", "dois itens", "um item" e "nenhum". Na questão 7, significam "mais de $8 \mathrm{~kg}^{\prime}$, " $8 \mathrm{~kg}^{\prime \prime}$, " $6 \mathrm{~kg}^{\prime}$ ", "4 kg" e "2 kg". Já na questão 8, se configuram por "mais de 10 por dia", 1 a 10 por dia", "nenhum nos últimos 6 meses", "nenhum no ano passado" $e$ "nenhum nos últimos 5 anos". A questão 9 apresenta apenas dois valores, 0 e 1, com significado de "algumas vezes e nunca", respectivamente. Na questão 10, os valores 0,1 , 2, 3 e 4 caracterizam-se por "quase diariamente", "com relativa frequência", "ocasionalmente", "quase nunca" e "nunca". Na questão 11, esses valores correspondem a "mais de 10 vezes por dia", "7 a 10 vezes por dia", " 3 a 6 vezes por dia", " 1 a 2 vezes por dia" e "nunca". O significado desses valores na questão 12 é: "mais de 20", "13 a 20", "11 a 12", "8 a 10" e "0 a 7". Na questão 13, os valores representam "quase diariamente", "com relativa frequência", "ocasionalmente", "quase nunca" e "nunca. A questão 14 também retrata dois valores apenas, sendo 0 equivalente a "algumas vezes", e 1 equivalente a "nunca". A questão 16 retrata os valores como "quase nunca", "raramente", "algumas vezes", "com relativa frequência" e "quase sempre". Na questão 19, os valores $0,1,2,3$ e 4 referem-se a "quase nunca", "raramente", "aloumas vezes", "com relativa frequência" e "quase sempre". Nas questões 20, 21, 23 e 24, os valores referem-se a "quase sempre", "com relativa frequência", "algumas vezes", "raramente" e "quase nunca". ** As colunas não totalizam 100\%, já que alguns alunos deixaram questões sem resposta. Fonte: Os autores. 


\section{DISCUSSÃO}

O maior número de mulheres cursando Medicina encontrado neste estudo está de acordo com os dados da população universitária brasileira registrados no Censo da Educação Superior do Brasil em 2014, que mostra que, do total de 7.828.013 matrículas, $56,66 \%$ são femininas ${ }^{16}$.

Quanto à distribuição étnica, a população foi constituída essencialmente por indivíduos que se autoclassificaram como de raça branca $(86,63 \%)$. Esta prevalência pode ser explicada por ser a Região Sul do Brasil marcada pela presença da colonização europeia, conforme indica o Instituto Brasileiro de Geografia e Estatística ${ }^{17}$.

Os resultados mostraram que $28,65 \%$ dos estudantes apontaram respostas indicativas de diagnóstico de depressão ou outra patologia crônica psiquiátrica, $26,56 \%$ fazem uso de medicamentos psiquiátricos e $55,38 \%$ relataram diagnóstico de depressão na família. A literatura mostra que os estudantes de Medicina são uma população vulnerável a essas patologias e outras que se correlacionam com estas, como abuso de álcool e uso de entorpecentes ${ }^{18}$. Porém, poucos estudantes de Medicina procuram ajuda quando estão com distúrbio psiquiátrico, pois temem o estigma associado à procura de ajuda e tratamento nestas situações.

Esses distúrbios psiquiátricos podem ser agravados quando os estudantes universitários precisam se afastar do aconchego familiar em decorrência da localização da universidade, pois o fato de o estudante dispor de pessoas próximas, com quem possa conversar e compartilhar sentimentos, é importante para retardar processos de estresse. Além disso, há um componente hereditário no transtorno de humor e depressivo, já que os parentes de primeiro grau de pessoas com tal enfermidade têm risco aumentado de desenvolver depressão de até três vezes quando comparados à população geral ${ }^{19}$.

As comparações entre estudos ainda são difíceis devido à escassez de trabalhos semelhantes, especialmente aqueles que comparam acadêmicos de Medicina no País, mesmo considerando o aumento recente do número de faculdades de Medicina no Brasil.

Quanto à classificação do estilo de vida dos acadêmicos, para Santos et al. ${ }^{20}$, é desejável que os indivíduos atinjam a classificação "bom". Nesse estudo, a maioria dos acadêmi$\cos (43,6 \%)$ foi classificada como "bom", ficando os alunos do quinto e sexto anos acima da média, classificados como "muito bom". Esse resultado pode ser explicado não só pela maturidade do acadêmico, desenvolvida ao longo da faculdade, mas também pelo senso de responsabilidade e manejo do seu tempo. O número de responsabilidades e deveres aumenta a cada ano, porém o estudante percebe a importância de cuidar de si mesmo antes de cuidar dos outros e aprende a inserir melhores práticas de vida em seu cotidiano. Um estudo realizado com internos do sexto ano de Medicina de universidades públicas e privadas de Santa Catarina constatou que metade dos acadêmicos realizava atividade física sistematizada, com frequência semanal. Além disso, apresentavam qualidade de vida positiva apesar dos níveis elevados de estresse a que eram submetidos ${ }^{21}$, demonstrando que mesmo com a elevada carga horária e tensão, os acadêmicos dos últimos anos tendem a ser mais conscientes em seus hábitos de vida.

Verificou-se que os acadêmicos pesquisados apresentam boa percepção sobre sua qualidade de vida. "Tenho alguém para conversar", "Consumo por semana" - relacionado à ingestão média de álcool por semana - e "Uso de cinto de segurança" são os itens que indicaram maior relação com estilo de vida saudável, os quais apresentaram pontuação relevante para a classificação desejável no EVF. Tal resultado é semelhante ao do estudo realizado na Universidade de Brasília, onde os acadêmicos apresentavam estrutura suficiente e eram capazes de criar melhores condições de vida em um curso que exige dedicação exclusiva ${ }^{12}$.

Embora os estudantes tenham pontuação baixa nas questões que refletem atividade física e diversão - "Sou vigorosamente ativo" e "Sou moderadamente ativo" -, isto não se mostrou um fator que inviabilizasse ou prejudicasse a conclusão do curso, mas são hábitos que influenciam a qualidade de vida. Além disso, "Aparento estar com pressa" foi um item que recebeu bastante pontuação, prejudicando, assim, o escore final do EVF. Outros fatores que diminuíram o escore final foram "Dirigir após a ingestão de bebidas alcoólicas", pois $41 \%$ dos acadêmicos relataram ter esse hábito "algumas vezes", e apenas 19\% quase sempre relaxam em seu tempo de lazer. A falta de prática de atividade física pode se dever à falta de horários compatíveis para frequentar academias em virtude da extensa carga horária do curso de Medicina ${ }^{3}$.

Quanto ao sono dos alunos, foi possível identificar que apenas 13\% têm uma boa noite de sono com frequência. Esse dado é relevante, pois um estudo mostrou que pode haver aumento do estresse subjetivo e humor negativo em resposta a estressores relativamente menores encontrados após uma noite de sono inadequada. Isso também pode demonstrar como a privação do sono contribui para a experiência das pessoas de "Sentir-se sobrecarregado" ou reagir excessivamente na presença de exigências cognitivas relativamente moderadas $^{22}$

Resultados semelhantes também foram vistos em estudo realizado no curso de Medicina de uma instituição do Estado 
de São Paulo, observando-se alta prevalência de distúrbios do sono e quase metade dos alunos sendo classificados com uma má qualidade de sono ${ }^{23}$.

Além disso, segundo Benedict et al. ${ }^{24}$, privação aguda do sono aumenta o processamento do estímulo hedônico no cérebro ligado ao desejo de consumir alimentos, independentemente dos níveis plasmáticos de glicose. Esse importante mecanismo pode estar relacionado aos resultados encontrados nesta pesquisa, com a maioria dos estudantes "quase nunca" a "algumas vezes" tendo uma alimentação saudável e, frequentemente, comendo em excesso alimentos ricos em açúcar, sal, gordura animal e bobagens e salgadinhos.

Dessa forma, a saúde mental do estudante é prejudicada, pois, mesmo em seu tempo de lazer e descanso, não se beneficia desses momentos e também não os aproveita de forma adequada. Apesar da boa classificação que os estudantes obtiveram no EVF, as questões aqui discutidas podem ser a razão para muitos estudantes se sentirem esgotados física e mentalmente, pois não descansam de forma satisfatória.

\section{CONCLUSÃO}

O presente estudo permitiu avaliar o estilo de vida geral de acadêmicos de Medicina com base no questionário autoaplicável "Estilo de Vida Fantástico", no qual foi obtida a classificação predominante "bom". Entretanto, fatores como sono e alimentação saudável não atingem níveis ideais nessa população, sendo a saúde negligenciada em detrimento das atividades acadêmicas.

Ressalta-se a importância de orientar os estudantes universitários para um estilo de vida mais saudável, com treinamento para o manejo de tempo, permitindo que conciliem as atividades acadêmicas com o cuidado com a saúde.

Apesar de os resultados serem satisfatórios, são necessários mais estudos sobre essa população, podendo haver continuidade desse acompanhamento do estilo de vida durante toda a graduação. Além disso, seria interessante realizar estudos sobre fatores que influenciam positiva e negativamente a vida acadêmica e como orientações sobre esses fatores poderiam ser efetivas para melhorar a qualidade de vida.

\section{REFERÊNCIAS}

1. Cardoso Filho FAB, Magalhães JF, da Silva KML, Pereira ISSD. Perfil do Estudante de Medicina da Universidade do Estado do Rio Grande do Norte (UERN). Rev. bras. educ. med. 2014; 39 (1) 32-40.

2. Feodrippe ALO, Brandão MCF, Valente TCO. Qualidade de Vida de Estudantes de Medicina:uma Revisão.Rev. bras. educ. med. 2013; 37(3) 418-428.
3. Figueiredo AM, Ribeiro GM, Reggiani ALM, Pinheiro BA, Leopoldo GO, Duarte JAH, et al. Percepções dos Estudantes de Medicina da UFOP sobre Sua Qualidade de Vida. Rev. bras. educ. med. 2014; 38 (4) 435-443.

4. Peres MFT, Barreto ADL, Babler F, Quaresma IYV, Arakaki JNL, Eluf-Neto J. Exposição à violência, qualidade de vida, depressão, e burnout entre estudantes de Medicina em uma universidade estadual paulista. RevMed 2014; 93 (3) $115-124$

5. Costa EFO, Santos SA, Santos ATRA, Melo EV, Andrade TM. Burnout Syndrome and associated factors among medical students: a cross-sectional study. Clin 2012; 67 (6) 573-579.

6. Almeida MAB de, Gutierrez GL, Marques R. Qualidade de vida: definição, conceitos e interfaces com outras áreas de pesquisa. EACH/USP2012.

7. Gonçalves A. Em busca do diálogo do controle social sobre o estilo de vida. In: Vilarta R, (org.) Qualidade de Vida e políticas públicas: saúde, lazer e atividade física. IPES 2004; 17-26.

8. World Health Organization. Health Promotion Glossary. Geneva; 1998.

9. Brito BJQ, Gordia AP, Quadros TMB. Lifestyle of college students: follow-up study during the first two years of the undergraduate. Med (RibPre Online) 2016; 49 (4) 293-302.

10. Gracino ME, Zitta ALL, Mangili OC, Massuda EM. A saúde física e mental do profissional médico: uma revisão sistemática. Saúde debate 2016; 40 (110) 244-263.

11. Della Santa N,CantilinoA. Suicídio entre Médicos e Estudantes de Medicina: Revisão de Literatura. Rev. bras. educ. med. 2016; 40 (4) 772-780.

12. Bampi LNS, Baraldi S, Guilhem D, Araújo MP, Campos ACO. Qualidade de Vida de Estudantes de Medicina da Universidade de Brasília. Rev. bras. educ. med. 2013; 37 (2) $217-225$

13. Martins GH, Martins RS, Prates MEF, Martins GC. Análise dos Parâmetros de Qualidade e Estilo De Vida de Universitários. Rev.Mack.Edu. Fís. Esp. 2012; 11(1) 22-30.

14. Rodriguez Anez CR, Reis RS,Petroski EL. Versão brasileira do questionário "estilo de vida fantástico": tradução e validação para adultos jovens. Arq. Bras.Cardiol. 2008; 91 (2) 102-109.

15. Pôrto EF, Kümpel C, Castro AAM, Oliveira IM, Alfieri FM. How life style has been evaluated: a systematic review. Acta Fisiátr. 2015; 22(4) 199-205.

16. Brasil. Instituto Nacional de Estudos e Pesquisas Educacionais Anísio Teixeira. Sinopses Estatísticas da Educação Superior: 2015. Brasília: MEC; 2016. 
17. Instituto Brasileiro de Geografia e Estatística (IBGE). Características étnico-raciais da população: Classificações e identidades 2013. Disponível em: https:/ /www.ibge.gov. br/estatisticas-novoportal/sociais/populacao/9372-caracteristicas-etnico-raciais-da-populacao.html.

18. Oliveira GS, Rocha CA, Santos BEF, Sena IS, Favaro L, Guerreiro MC. Prevalência e fatores associados à depressão em estudantes de Medicina da Universidade Federal do Amapá. Rev. Med. Saú. Bras. 2016; 5 (3) p.186-199.

19. Torres AR, Oliveira AM, Azevedo AP, Floresi ACF, West ACA, Kleinman A, et al. Compêndio de Clínica Psiquiátrica. São Paulo: Manole; 2012. 296-314.

20. Santos JJA, Saracini N, da Silva WC, Guilherme JH,da Costa TA, Silva MRAG. Estilo de vida relacionado à saúde de estudantes universitários: comparação entre ingressantes e concluintes. Abcs HealScien 2014; 39 (1) 17-23. http:// dx.doi.org/10.7322/abcshs.v39i1.256.

21. Meyer C, Guimarães ACA, Machado Z, Parcias SR. Qualidade de vida e Estresse ocupacional em estudantes de Medicina. Rev. bras. educ. med. 2012; 36 (4) 489-498.

22. Minkel JD, Banks S, Htaik O, Moreta MC, Jones CW, McGlinchey EL, Simpson NS, Dinges DF. Sleep deprivation and stressors: Evidence for elevated negative affect in response to mild stressors when sleep deprivedEmotion. 2012; 12(5) 1015-20. doi: 10.1037/a0026871

23. Moraes CAT, Edelmuth DGL, Novo NF, Hübner CVK. Qualidade de sono em estudantes de Medicina do método de aprendizado baseado em problemas. RevFacMedRibPre e HospClín da Fmrp 2013; 46, (4) 389-937.

24. Benedict C, Brooks SJ, O'Daly OG, Almèn MS, Morell A, Åberg K et al. Acute Sleep Deprivation Enhances the Brain's Response to Hedonic Food Stimuli: An fMRI Study. J ClinEndocrinolMetab2012; 97(3) 443-7. doi: 10.1210/ jc.2011-2759.

\section{CONTRIBUIÇÃO DOS AUTORES}

Todos os autores contribuíram igualmente na elaboração do artigo, responsáveis pelo estudo em todas as suas fases, desde a concepção inicial até a execução, coleta de dados, análise dos resultados e redação final.

\section{CONFLITO DE INTERESSES}

Os autores declaram não haver conflito de interesse.

\section{ENDEREÇO PARA CORRESPONDÊNCIA}

Ana Carolina Tomiyoshi

Instituição vinculada: Centro Universitário de Maringá - UniCesumar

Endereço: Av. Guedner, 1610 - Zona 08, Maringá - PR CEP:87050-900

E-mail: carol_.tomiyoshi@hotmail.com 